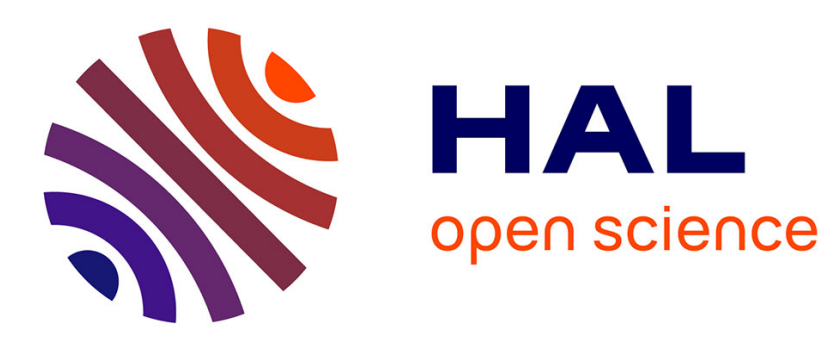

\title{
Amorphisation d'une cible d'AsGa par des ions As
}

J. Beauvillain, Alain Claverie, K. Akmoum

\section{To cite this version:}

J. Beauvillain, Alain Claverie, K. Akmoum. Amorphisation d'une cible d'AsGa par des ions As. Journal de Physique III, 1992, 2 (3), pp.407-414. 10.1051/jp3:1992138 . jpa-00248754

\section{HAL Id: jpa-00248754 https://hal.science/jpa-00248754}

Submitted on 1 Jan 1992

HAL is a multi-disciplinary open access archive for the deposit and dissemination of scientific research documents, whether they are published or not. The documents may come from teaching and research institutions in France or abroad, or from public or private research centers.
L'archive ouverte pluridisciplinaire HAL, est destinée au dépôt et à la diffusion de documents scientifiques de niveau recherche, publiés ou non, émanant des établissements d'enseignement et de recherche français ou étrangers, des laboratoires publics ou privés. 
Classification

Physics Abstracts

$61.70 \mathrm{~T}-61.80 \mathrm{~J}-72.68$

\title{
Amorphisation d'une cible d'AsGa par des ions As
}

\author{
J. Beauvillain, A. Claverie et K. Akmoum \\ CEMES-LOE/CNRS, B.P. 4347, 31055 Toulouse, Cedex, France
}

(Reçu le 25 juillet 1991, révisé et accepté le 7 novembre 1991)

\begin{abstract}
Résumé. - Le modèle de la densité d'énergie critique donne une analyse pratique de la transition cristal/amorphe induite par implantation ionique. Nous l'avons appliqué au cas d'un cristal d'arséniure de gallium irradié, à température ambiante, par des ions arsenic accélérés sous une tension de $100 \mathrm{kV}$. L'observation des dépôts irradiés en microscopie électronique par la tranche nous a permis de tracer un diagramme d'état expérimental qui permet pour un tel système de prédire les effets d'une implantation. En associant ces résultats à la distribution de la densité d'énergie de dommage donnée par TRIM, nous avons trouvé pour ce système une valeur de la densité d'énergie critique égale à $50 \mathrm{eV} / \mathrm{mol}$.

Abstract. - The «critical damage energy density » model gives a practical analysis of the amorphous-crystalline ion induced transition, Cross-sectional electron microscopy and related diffraction techniques have been applied to the characterization of arsenide-bombardement induced amorphization of gallium arsenide at room temperature. Combining the experimental measurements of the extension of the amorphous layer for increasing doses with concepts arising from the «critical damage energy density » model leads to $E_{\mathrm{dc}}$ values of about $50 \mathrm{eV} \mathrm{mol} .^{-1}$ for $100 \mathrm{keV}$ arsenide, for the cristalline to amorphous transformation to occur.
\end{abstract}

\section{Introduction.}

L'implantation ionique est une technique très utilisée dans les traitements de surfaces ou dans la réalisation de composants électroniques. Outre l'apport des ions dans le matériau, cette technique génère aussi des défauts cristallographiques qui peuvent provoquer dans le solide implanté une transition de l'état cristallin vers l'état amorphe. Cet aspect destructeur de la périodicité peut être très utile : la préamorphisation d'un matériau semiconducteur avant dopage permet par exemple de supprimer les effets de canalisation et l'implantation s'effectue alors de façon plus homogène.

Le but de cette étude sur la transition cristal-amorphe induite par implantation dans l'arséniure de gallium $\langle 100\rangle$ est de pouvoir posséder un outil permettant de prévoir les régions du substrat amorphisées en fonction des doses d'ions arsenic implantés avec des énergies de 100 et $150 \mathrm{keV}$.

\section{Analyse et méthodologie.}

1.1 InTERPRÉTATION DU PHÉNOMÈnE. - Le modèle de la densité d'énergie critique est le plus utile pour pouvoir prévoir et décrire l'amorphisation d'une cible cristalline par 
implantation ionique [1-5]. Bien que macroscopique et non réaliste au stade de l'interaction individuelle, ce modèle a le mérite d'être efficace et simple d'emploi.

Il prend pour hypothèse qu'un cristal transite spontanément vers l'amorphe dès qu'il reçoit, par interaction nucléaire, une densité d'énergie critique $: E_{\mathrm{dc}}$. Cette transition cristal/amorphe peut être schématisée sur le diagramme de configuration de la figure 1 . Ce modèle présuppose que le cristal endommagé passe par un état saturé en défauts avant de relaxer vers l'état amorphe thermodynamiquement plus stable.

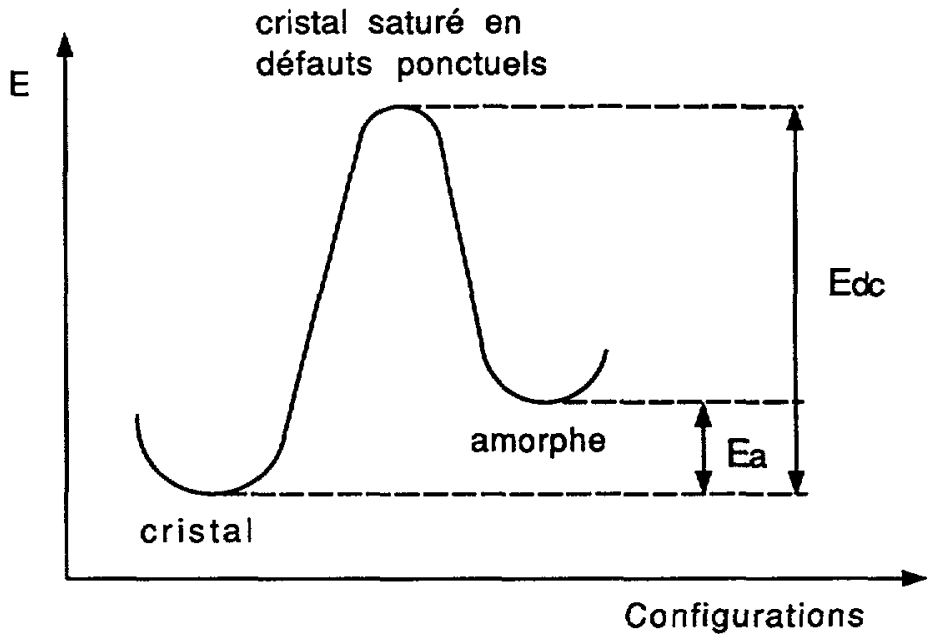

Fig. 1. - Schéma de la transition cristal-amorphe.

[Amorphous-crystalline transition illustration.]

1.2 UTILISATION DU MODĖle. - Pour prédire l'apparition et l'extension des couches amorphes générées dans l'arséniure de gallium lors de l'implantation d'ions arsenic, il est nécessaire de connaître deux données :

- la distribution, en fonction de la profondeur, de la densité d'énergie de dommage $E_{\mathrm{d}}$ déposée, par interactions nucléaires, dans les matériaux (Fig. 2);

- la valeur de la densité d'énergie critique du matériau : $E_{\mathrm{dc}}$.

D'après le modèle de la densité d'énergie critique, dès que $E_{\mathrm{d}}$ est supérieur à $E_{\text {dc }}$ alors le substrat transite du cristal vers l'amorphe.

Une autre manière de représenter l'extension des couches amorphes en fonction des doses croissantes est schématisée sur la figure 3. C'est une représentation sous forme d'un diagramme d'état dose-profondeur où la courbe délimite les deux phases.

Pour un système donné (cible, ions, énergie, flux, température...). Cette courbe permet de localiser les régions amorphes dès que l'on connaît la dose implantée, ce qui est le but de cette étude.

1.3 DÉtermination DE LA DENSITÉ D'ÉNERGIE CRITIQUe $E_{\mathrm{dc}}$ - Le diagramme d'état (Fig. 3) permet aussi, pour une distribution de la densité d'énergie déposée donnée, d'avoir accès à la valeur de la densité.

En effet, pour une distribution d'énergie de dommage (Fig. 2) donnée, suivant la valeur de $E_{\mathrm{dc}}$, la courbe d'énergie critique $E_{\mathrm{dc}}[5,6]$ de la figure 3 se déplace le long de l'axe vertical. Un diagramme d'état dose/profondeur expérimental imposera donc une valeur de $E_{\mathrm{dc}}$. 


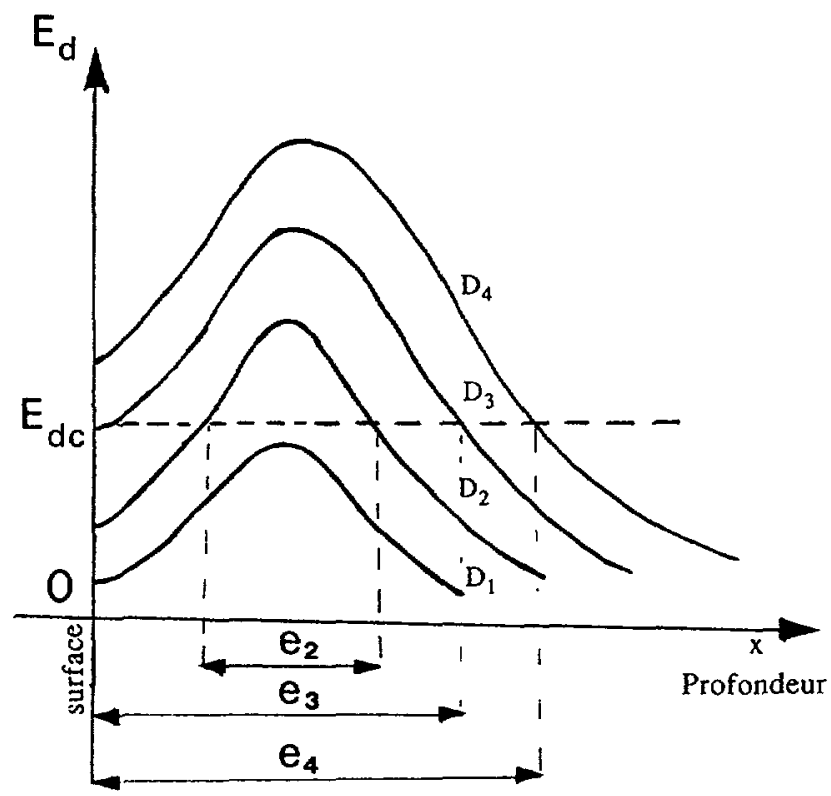

Fig. 2. - Distribution de la densité d'énergie de dommage en fonction de la profondeur pour des doses implantées croissantes $\left(D_{4}>D_{3}>D_{2}>D_{1}\right)$. Les longueurs $e_{2}, e_{3}$, et $e_{4}$ représentent les épaisseurs et la localisation de l'amorphe dans la cible $\left(e_{1}=0\right)$.

[Damage energy depth distribution for increasing doses $\left(D_{4}>D_{3}>D_{2}>D_{1}\right)$. The lengths, $e_{1}$, $e_{2}, e_{3}, e_{4}$ give thickness and localization of amorphous layers $\left(e_{1}=0\right)$.]

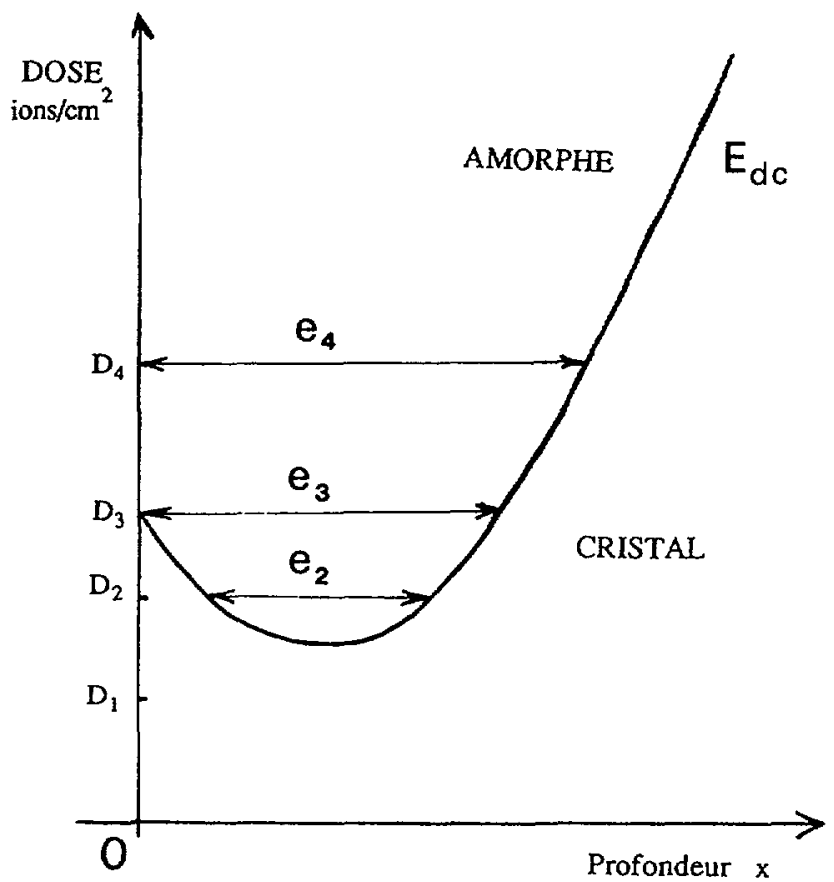

Fig. 3. - Diagramme d'état dose/profondeur.

[Dose/Depth state diagram.] 
De nombreux auteurs, par différents travaux expérimentaux, ont essayé de déterminer $E_{\mathrm{dc}}$ pour un certain nombre de matériaux, les plus sollicités furent évidemment les semiconducteurs. Pour l'arséniure de gallium, on peut citer Sadana et al. [4] qui donnent, dans le cas d'ions $S e / 450 \mathrm{keV}$, une valeur de $E_{\mathrm{dc}}$ égale à $11 \mathrm{eV} / \mathrm{mol}$. à température ambiante, Gibbons et al. [7] qui rapportent une valeur de $E_{\mathrm{dc}}$ comprise entre 50 et $77 \mathrm{eV} / \mathrm{mol}$. pour une implantation effectuée, avec des ions $\mathrm{Si} / 100 \mathrm{keV}$, à la température de l'azote liquide, Wesch et al. [8] qui trouvent deux valeurs : l'une à basse température entre 11 et $13 \mathrm{eV} / \mathrm{mol}$. et l'autre à température ambiante entre 38 et $46 \mathrm{eV} / \mathrm{mol}$. pour des ions $\mathrm{Ar} / 200 \mathrm{keV}$. Plus récemment, toujours pour l'arséniure de gallium, Taniwaki et al. [9] donnent des valeurs variant de 1,5 à $50 \mathrm{eV} / \mathrm{mol}$. à la température ambiante avec des ions $\mathrm{Sn} / 100 \mathrm{keV}$.

La disparité des résultats provient de la variété des systèmes étudiés. Ayant accès à un diagramme dose-profondeur expérimental pour les systèmes (As/100 keV/AsGa) et (As/150 keV/AsGa) nous en déduirons les valeurs de $E_{\mathrm{dc}}$ pour ces deux systèmes particuliers.

\section{Diagramme d'état dose/profondeur expérimental.}

Pour le système (As/100 keV/AsGa) nous obtenons le diagramme d'état par une mesure directe des couches amorphes en microscopie électronique à transmission en effectuant des coupes transversales des cibles implantées $[5,6,10]$. Les échantillons sont nettoyés, implantés, puis amincis avant d'être observés au microscope.

L'implantation est effectuée sur des cibles d'arséniure de gallium $\langle 100\rangle$ monocristallin, semi-isolant, non dopé, de dimensions $4 \times 3 \times 0,5 \mathrm{~mm}^{3}$ obtenues par découpe d'une plaquette de $0,5 \mathrm{~mm}$ d'épaisseur présentant une face $\langle 100\rangle$ polie.

En cours d'implantation le porte-objet est incliné de façon à minimiser les effets de la canalisation. L'isotope implanté est l'arsenic 75, il est accéléré sous une tension de $100 \mathrm{keV}$. Le flux ionique est maintenu sensiblement égal à $1 \mu \mathrm{A} / \mathrm{cm}^{2}$. Divers échantillons ont été implantés, à température ambiante, avec des doses croissantes variant de $10^{13}$ à $10^{15}$ ions $/ \mathrm{cm}^{2}$.

La figure 4 présente les clichés de microscopie électronique par transmission des divers échantillons observés.

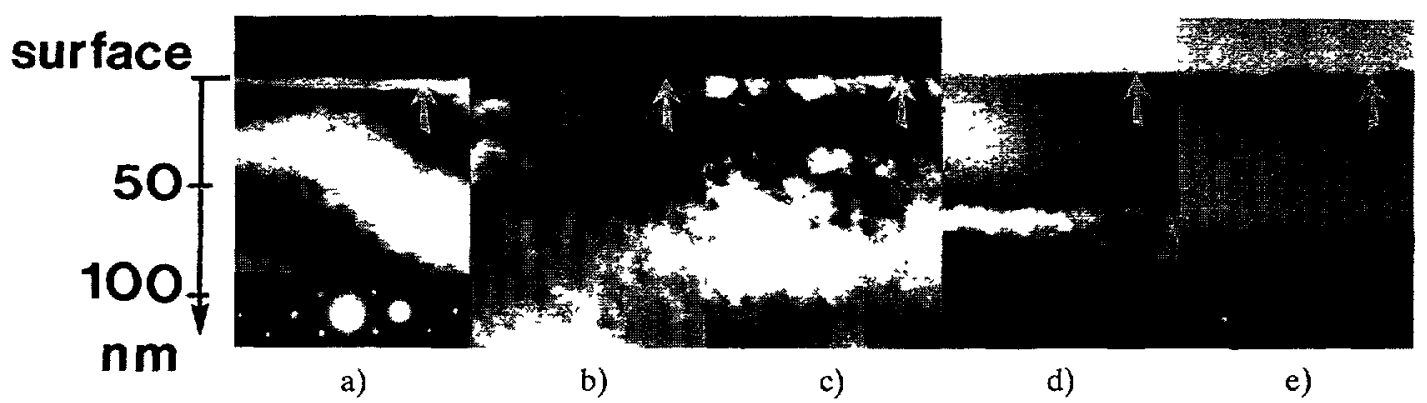

Fig. 4. - Formation et extension d'une couche amorphe dans l'arséniure de gallium par implantation As, à $100 \mathrm{keV}$, à température ambiante pour des doses croissantes. a) image en champ sombre pour une dose de $1 \times 10^{13}$ ions $/ \mathrm{cm}^{2}$. La cible reste cristalline; b) image en champ sombre pour une dose de $5 \times 10^{13}$ ions $/ \mathrm{cm}^{2}$. Apparition de « poches » amorphes; c) image en champ sombre pour une dose de $1 \times 10^{14}$ ions $/ \mathrm{cm}^{2}$. Création d'une couche amorphe continue sous la surface; d) image en champ clair pour une dose de $5 \times 10^{14}$ ions $/ \mathrm{cm}^{2}$. La couche amorphe émerge en surface; e) image en champ clair pour une dose de $1 \times 10^{15}$ ions $/ \mathrm{cm}^{2}$. La couche amorphe s'étend à l'intérieur du matériau.

[XTEM micrographs showing the creation and the extension of an amorphous layer due to arsenide implantation at $100 \mathrm{keV}$ into gallium arsenide at room temperature. Dose rate $=1 \mu \mathrm{A} / \mathrm{cm}^{2} ;$ a) $10^{13}$ ions $/ \mathrm{cm}^{2}$; b) $5 \times 10^{13}$ ions $/ \mathrm{cm}^{2}$; c) $10^{14}$ ions $/ \mathrm{cm}^{2}$; d) $5 \times 10^{14}$ ions $/ \mathrm{cm}^{2}$; e) $10^{15}$ ions $/ \mathrm{cm}^{2}$.] 
L'ensemble des mesures expérimentales obtenues à partir des clichés de la figure 4 est donné par la figure 5.

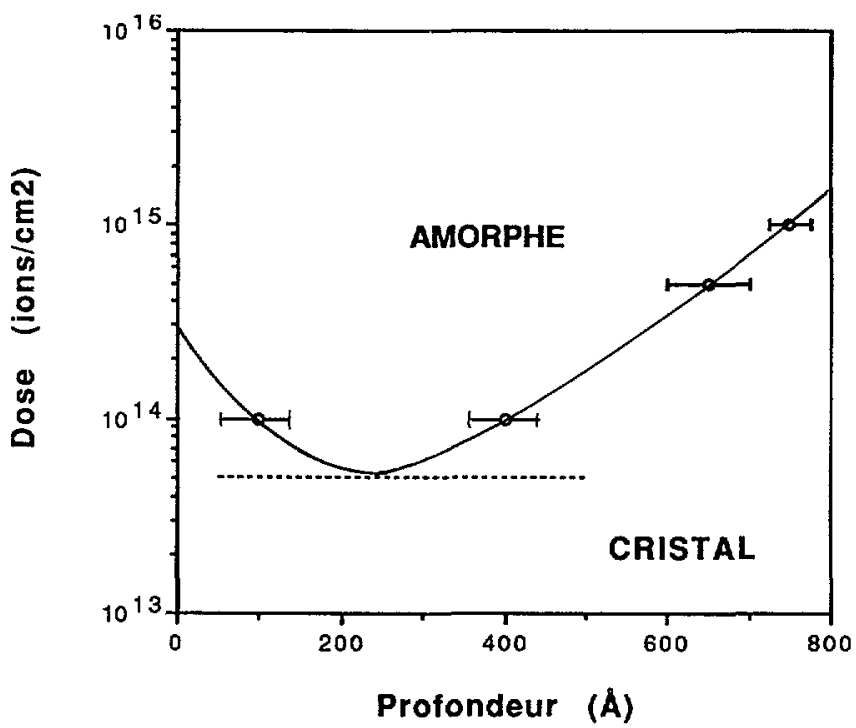

Fig. 5. - Courbe expérimentale représentant un diagramme d'état dose/profondeur pour le système As à $100 \mathrm{keV}$ dans le GaAs à température ambiante.

[Experimental dose/depth state diagram for the $100 \mathrm{keV}$ As-AsGa room temperature, $1 \mu \mathrm{A} / \mathrm{cm}^{2}$ system.]

Les barres expérimentales dans la direction de l'axe des abscisses représentent la rugosité de l'interface cristal/amorphe pour chaque dose implantée. Les pointillés sont la représentation d'une distribution discontinue de «poches » amorphes.

Selon nos résultats expérimentaux, la dose minimale qui crée une couche amorphe continue en profondeur dans l'arséniure de gallium est comprise entre $5 \times 10^{13}$ et $10^{14}$ ions $/ \mathrm{cm}^{2}$. Par contre, la dose minimale d'amorphisation en surface est contenue dans l'intervalle de doses $\left[10^{14}\right.$ à $\left.5 \times 10^{14}\right]$ ions $/ \mathrm{cm}^{2}$. La courbe de la figure 5 permet donc de prévoir, l'amorphisation induite par implantation d'ions arsenic de $100 \mathrm{keV}$ dans de l'arséniure de gallium à température ambiante avec un flux ionique correspondant à une densité de courant de $1 \mu \mathrm{A} / \mathrm{cm}^{2}$.

\section{Détermination de la densité d'énergie critique : $\boldsymbol{E}_{\mathrm{dc}}$.}

Avec la courbe expérimentale de la figure 5 il est possible de déterminer la valeur de $E_{\mathrm{dc}}$ dès que l'on est en possession d'une distribution en profondeur de la densité d'énergie de dommage (Fig. 2). Cette distribution nous est fournie par le calcul TRIM [11] et nous donne, pour un ion arsenic accéléré à $100 \mathrm{keV}$, implanté dans une cible d'arséniure de gallium, les valeurs reportées sur la figure 6 .

Selon ce calcul théorique, l'amorphe, dû au bombardement ionique, doit commencer à apparaître à $26 \mathrm{~nm}$ sous la surface et déboucher en surface dès que son épaisseur atteint environ $60 \mathrm{~nm}$.

Ces résultats concordent avec les résultats expérimentaux précédents (Fig. 5). A l'aide de la courbe de la figure 6 , il est possible de construire pour une valeur de $E_{\mathrm{dc}}$, un diagramme dose/profondeur. 


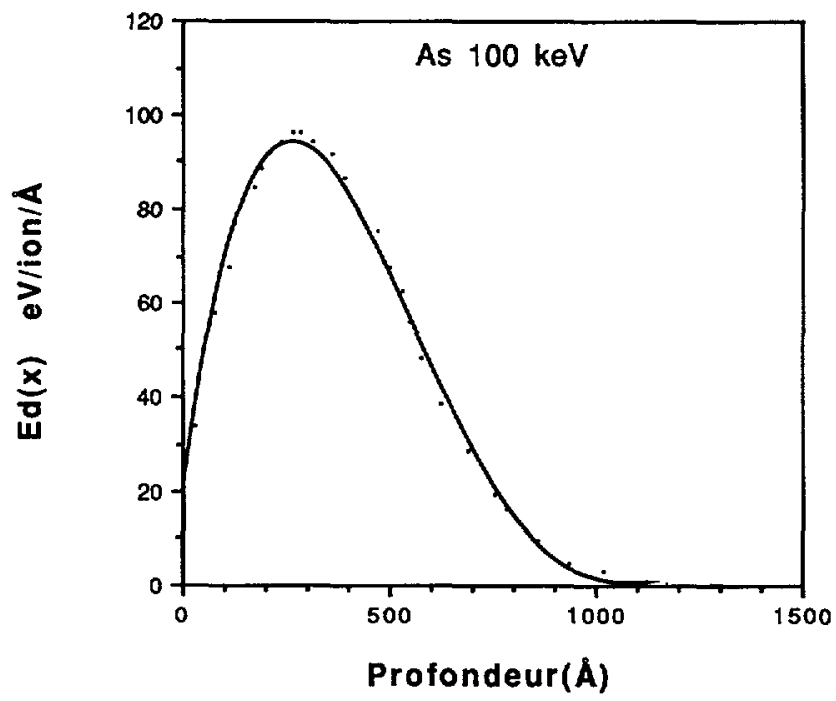

Fig. 6. - Distribution en profondeur de la densité d'énergie de dommage déposée par un ion As de $100 \mathrm{keV}$ dans le GaAs.

[Damage energy density distribution for one ion $100 \mathrm{keV}$ As-AsGa system.]

Sur la figure 7 , nous présentons les diagrammes obtenus pour $E_{\mathrm{dc}}$ égale à 10 , 30 et $70 \mathrm{eV} / \mathrm{mol}$. Les points expérimentaux obtenus nous permettent d'estimer, pour le système que nous considérons (As ; $100 \mathrm{keV} ; \mathrm{AsGa} ; 1 \mu \mathrm{A} / \mathrm{cm}^{2}$; température ambiante), la valeur de

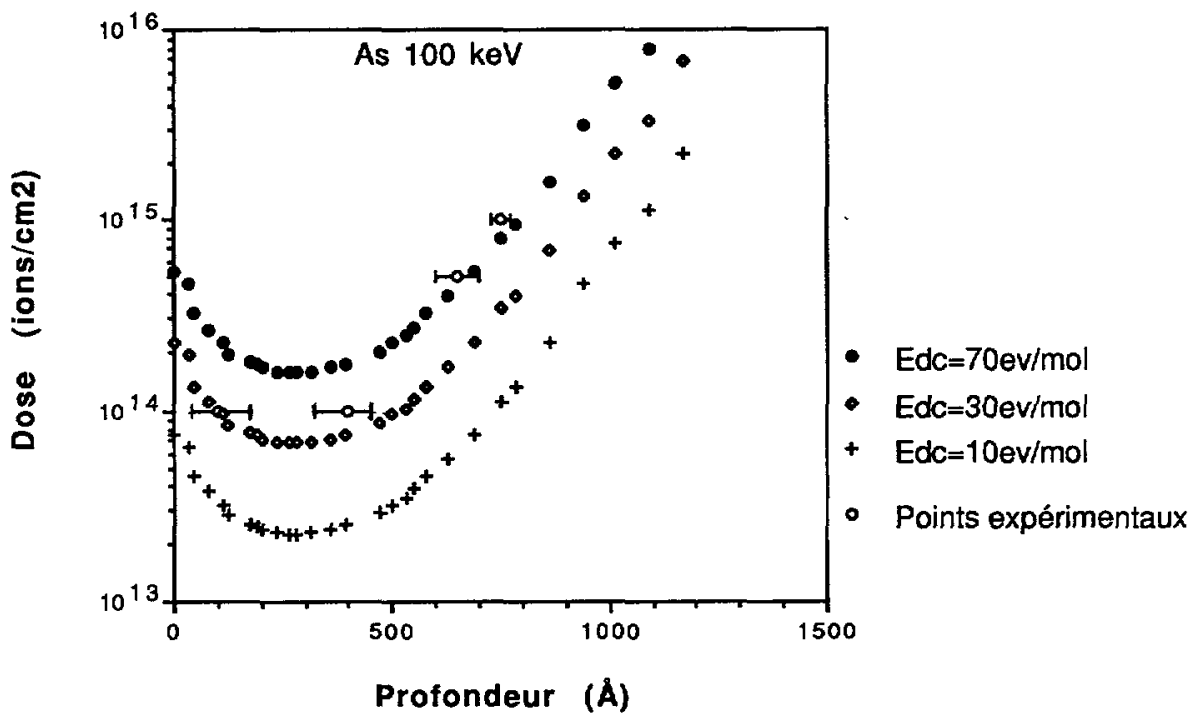

Fig. 7. - Confrontation des résultats expérimentaux au calcul théorique de la distribution de la densité d'énergie de dommage pour différentes valeurs de $E_{\mathrm{dc}}$, dans le cas du système As, $100 \mathrm{keV}$ dans le GaAs.

[Dose/depth state diagram for the $100 \mathrm{keV}$ As-AsGa, room temperature, $1 \mu \mathrm{A} / \mathrm{cm}^{2}$ system. Critical damage energy density model assuming three different critical damage energy density values for the c/a transformation. XTEM measurements in bars and rings.] 
$E_{\mathrm{dc}}$ à $50 \mathrm{eV} / \mathrm{mol}$. (soit $11 \times 10^{23} \mathrm{eV} / \mathrm{cm}^{3}$ ) ou plus exactement à une valeur comprise entre 30 et $70 \mathrm{eV} / \mathrm{mol}$. (soit 6,5 et $15 \times 10^{23} \mathrm{eV} / \mathrm{cm}^{3}$ ).

\section{Discussion.}

4.1 VAlidité DU MODÈlE. - La difficulté de donner une valeur précise pour la densité d'énergie critique, après confrontation des résultats expérimentaux aux prévisions théoriques, pourrait venir du fait que le modèle, facilité d'utilisation oblige, n'est qu'une approche macroscopique de la réalité.

Une interprétation plus réaliste du phénomène, nous semble-t-il, a été proposée par Taniwaki et al. [9] qui étudient l'amorphisation du GaAs $\langle 111\rangle$ par des ions $\mathrm{Sn}_{+}$de $100 \mathrm{keV}$ à température ambiante avec un flux de $4,5 \times 10^{13} \mathrm{ions} / \mathrm{cm}^{2} / \mathrm{s}$. Ces auteurs suggèrent l'existence de deux modes d'amorphisation, soit deux valeurs de $E_{\mathrm{dc}}$. L'une correspondrait à des doses relativement faibles quand une couche amorphe homogène et continue n'est pas encore formée, dans notre cas elle correspondrait à des doses inférieures à $10^{14}$ ions $/ \mathrm{cm}^{2}$ et vaudrait $30 \mathrm{eV} / \mathrm{mol}$. L'autre correspondrait à des doses plus élevées et serait celle nécessaire à l'extension de l'amorphe quand une couche continue est créée, dans notre cas elle correspondrait à des doses supérieures ou égales à $5 \times 10^{14}$ ions $/ \mathrm{cm}^{2}$ et serait égale à $70 \mathrm{eV} / \mathrm{mol}$. En effet, dans le deuxième cas, la transition cristal-amorphe s'opère non seulement grâce au processus d'accumulation du dommage mais aussi par une action de l'interface cristal-amorphe ce qui justifierait une valeur de $E_{\mathrm{dc}}$ différente, plus grande dans notre cas. Taniwaki et al. [9], pour un tout autre système, avec un flux plus élevé, ont trouvé un effet totalement contraire.

4.2 EFFET DE L'ÉNERGIE. - Afin de voir si l'énergie des ions incidents a un effet pertinent nous avons refait la même étude dans les mêmes conditions expérimentales mais avec des ions arsenic de $150 \mathrm{keV}$. Les points expérimentaux obtenus nous donnent une valeur de $E_{\mathrm{dc}}$ égale à $100 \mathrm{eV} / \mathrm{mol}$. pratiquement le double de celle obtenue ci-dessus.

A température ambiante, à flux fixe, la densité d'énergie critique $E_{\mathrm{dc}}$ n'est donc pas une caractéristique intrinsèque de l'arséniure de gallium.

\section{Conclusion.}

Le modèle de la densité d'énergie critique permet de décrire la transition cristal-amorphe dans l'arséniure de gallium. Pour utiliser ces résultats et pouvoir prédire les effets d'une implantation d'ions arsenic il sera nécessaire de prendre la distribution en densité d'énergie de dommage donnée par TRIM et les valeurs de densité d'énergie critique de $50 \mathrm{eV} / \mathrm{mol}$. pour les ions arsenic de $100 \mathrm{keV}$ et de $100 \mathrm{eV} / \mathrm{mol}$. pour les ions As de $150 \mathrm{keV}$. Les paramètres température et flux sont aussi à considérer, seules des implantations à très basses températures pourraient, peut-être, nous donner accès à une valeur de $E_{\mathrm{dc}}$ qui correspondrait à une caractéristique intrinsèque de l'arséniure de gallium. Les résultats trouvés permettent cependant de prévoir les couches amorphes induites pour les systèmes considérés ou de donner un ordre de grandeur pour tout système analogue. 


\section{Bibliographie}

[1] Stein H. J., Vook F. L., Brice D. K., Borders J. A. et Picraux S. T., Proc. 1st Int. Conf. on Ion implantation, L. T. Chadderton and F. H. Eisen Eds. 17 (Gordon and Breach London 1971).

[2] Narayan J., Fathy D., Oen O, S. and Holland O. W., J. Vac. Sci. Technol. A 2 (3) (1984).

[3] Sands T., Sadana D. K., Gronsky R. and Washburn J., Appl. Phys. Lett. 44 (1984) 874.

[4] Sadana D. K., Nucl. Instrum. Methods B 78 (1985) 375-386.

[5] Claverie A., Roumili A., Gessinn N. and Beauvillain J., Mater. Sci. Eng. B 4 (1989) 205-209.

[6] Claverie A., Vieu C., Faure J. and Beauvillain J., Mater. Sci. Eng. B 2 (1989) 99.

[7] Opyd W. G., Gibbons J. F., Bravma J. C. and Parker M. A., Appl. Phys, Lett. 49 (1986) 974.

[8] Wesch W., Wendler E., Gotz G. and Kekelidse N. P., J. Appl. Phys. 65 (1989) 519.

[9] Taniwaki M., Koide H., Yoshimoto N., Yoshire T., Ohnuki S., Maeda M. and Sassa K., $J$. Appl. Phys. 67 (1990) 4036.

[10] Claverie A., Vieu C., Faure J. and Beauvillain J., J. Appl. Phys. 64 (1988) 4415.

[11] Zigler J. F., Biersack J. P. and LitTMARK U., The stopping and range of ions in solids (Pergamon Press New York, 1985). 